\title{
Airy Kernel and Painlevé II
}

\author{
Craig A. Tracy \\ Department of Mathematics \\ and \\ Institute of Theoretical Dynamics \\ University of California \\ Davis, CA 95616, USA \\ email address: tracy@itd.ucdavis.edu \\ Harold Widom \\ Department of Mathematics \\ University of California \\ Santa Cruz, CA 95064, USA \\ email address: widom@math.ucsc.edu
}

\begin{abstract}
We prove that the distribution function of the largest eigenvalue in the Gaussian Unitary Ensemble (GUE) in the edge scaling limit is expressible in terms of Painlevé II. Our goal is to concentrate on this important example of the connection between random matrix theory and integrable systems, and in so doing to introduce the newcomer to the subject as a whole. We also give sketches of the results for the limiting distribution of the largest eigenvalue in the Gaussian Orthogonal Ensemble (GOE) and the Gaussian Symplectic Ensemble (GSE). This work we did some years ago in a more general setting. These notes, therefore, are not meant for experts in the field.
\end{abstract}

\section{Introduction}

For the finite $N$ random matrix models GOE, GUE and GSE, the probability that no eigenvalues lie in a set $J, E_{\beta}(0 ; J)$, equals a Fredholm determinant of an integral operator with scalar kernel for GUE $(\beta=2)$ and equals the square root of a Fredholm determinant of an integral operator with matrix kernel for $\operatorname{GOE}(\beta=1)$ and $\operatorname{GSE}(\beta=4)$. Gaudin 17 (using Mehta's newly invented method of orthogonal polynomials [33]) first discoverd this connection between random matrix theory and Fredholm determinants, for GUE in the bulk scaling limit, in 1961. In 1970 Dyson [15] showed that the $n$-point correlations for circular ensembles are expressible as quaternion determinants. The next year Mehta [34] extended Dyson's formalism to include the GOE and the GSE. From these representations of the $n$ point correlations, one deduces that $E_{\beta}(0 ; J)^{2}(\beta=1,4)$ are Fredholm determinants. Later several authors generalized this construction to other invariant ensembles. See [46, 51 for simplified proofs of these facts. 


\section{Distribution of Largest Eigenvalue, $\beta=2$}

\subsection{Fredholm Determinant Representation}

In finite $N$ GUE (see, e.g., [35, 46])

$$
E_{N, 2}(0 ; J)=\operatorname{det}\left(I-K_{N, 2}\right)
$$

where $K_{N, 2}$ is the integral operator

$$
\begin{aligned}
\left(K_{N, 2} f\right)(x) & =\int_{J} K_{N, 2}(x, y) f(y) d y, \\
K_{N, 2}(x, y) & =\frac{\varphi(x) \psi(y)-\psi(x) \varphi(y)}{x-y}, \\
\varphi(x) & =\left(\frac{N}{2}\right)^{1 / 4} \varphi_{N}(x), \\
\psi(x) & =\left(\frac{N}{2}\right)^{1 / 4} \varphi_{N-1}(x)
\end{aligned}
$$

and the $\varphi_{k}(x)$ are the harmonic oscillator wave functions

$$
\varphi_{k}(x)=\frac{1}{\sqrt{2^{k} k ! \sqrt{\pi}}} e^{-x^{2} / 2} H_{k}(x) .
$$

(The $H_{k}(x)$ are the Hermite polynomials and the sequence $\left\{\varphi_{k}\right\}_{k \geq 0}$ forms a complete orthonormal sequence in $L^{2}(\mathbf{R})$.)

For $J=(t, \infty)$, the probability that no eigenvalues lies in $J$ is the same as the probability that all eigenvalues lie to the left of $t$; and in particular, that the largest eigenvalue, $\lambda_{\max }(N)$, lies to the left of $t$ :

$$
F_{N, 2}(t):=\operatorname{Prob}\left(\lambda_{\max }(N)<t\right)=E_{2}(0 ;(t, \infty)) .
$$

(Since the distribution functions all have continuous densities, we need not worry about the difference between $<$ and $\leq$.)

\section{$2.2 \quad$ Edge Scaling Limit}

Fixing $t$, Prob $\left(\lambda_{\max }(N)<t\right) \rightarrow 0$ as $N \rightarrow \infty$ since the largest eigenvalue increases with $N$; and hence, the probability that it remains less than any fixed number tends to zero. To obtain, therefore, a nontrivial limiting distribution we must introduce a normalized random variable. Such situations are common in probability theory, e.g. the central limit theorem.

The density of eigenvalues at $x, \rho(x)$, equals $K_{N, 2}(x, x)$. Replacing $K_{N, 2}(x, y)$ by

$$
\frac{1}{\rho(z)} K_{N, 2}\left(z+\frac{x}{\rho(z)}, z+\frac{y}{\rho(z)}\right)
$$

has the effect of making the point $z$ in the spectrum the new origin and rescaling so that the eigenvalue density at this point becomes equal to 1 . The "edge of the spectrum" corresponds 
to $z \sim \pm \sqrt{2 N}$. (The constant $\sqrt{2}$ results from the choice of the standard deviation of the matrix elements in GUE - here we choose a standard deviation equal to $1 / \sqrt{2}$ which is the same as in Mehta [35].) The edge scaling limit [10, 16] for the kernel is then

$$
\lim _{N \rightarrow \infty} \frac{1}{2^{1 / 2} N^{1 / 6}} K_{N, 2}\left(\sqrt{2 N}+\frac{x}{2^{1 / 2} N^{1 / 6}}, \sqrt{2 N}+\frac{y}{2^{1 / 2} N^{1 / 6}}\right)=K_{\text {Airy }}(x, y)
$$

where $K_{\text {Airy }}$ has the form (1) with

$$
\varphi(x)=\operatorname{Ai}(x), \quad \psi(x)=\operatorname{Ai}^{\prime}(x),
$$

and $\operatorname{Ai}(x)$ the Airy function. Given $t$ and $N$, we define $s$ by

$$
t=\sqrt{2 N}+\frac{s}{2^{1 / 2} N^{1 / 6}} .
$$

Using (4) and the Fredholm expansion of $\operatorname{det}\left(I-K_{N, 2}\right)$, it follows as $N \rightarrow \infty$ and $t \rightarrow \infty$ such that $s$ is fixed that (the edge scaling limit)

$$
\lim F_{N, 2}(t)=F_{2}(s)=\operatorname{det}\left(I-K_{\text {Airy }}\right)
$$

where $K_{\text {Airy }}$ acts on $L^{2}((s, \infty))$.

Remarks: Inspecting (2) and (3) we see that one needs asymptotic formulas for the Hermite polynomials $H_{k}(x)$ for large values of both $x$ and $k$. Fortunately, such formulas are known and were derived by Plancherel and Rotach in 1929. (They can be found in the Bateman Manuscripts.) Using these asymptotic formulas it is then straighforward to derive (4). Recent progress has been made in deriving analogous Plancherel-Rotach type formulas for orthogonal polynomials whose weight function is of the form $e^{-V}$ [8, 13]. The proofs are considerably more difficult than in the classical Hermite case. These formulas then allow one to prove that the Airy kernel arises in the edge scaling limit under much more general circumstances.

\subsection{Painlevé II Representation for $F_{2}$}

For notational convenience we denote $K_{\text {Airy }}$ by $K$ in this subsection. It will also be convenient to think of our operator $K$ as acting, not on $(s, \infty)$, but on $\mathbf{R}$ and to have kernel

$$
K(x, y) \chi(y)
$$

where $\chi$ is the characteristic function of $J$. Since the integral operator $K$ is trace-class and depends smoothly on the parameter $s$, we have the well known formula

$$
\frac{d}{d s} \log \operatorname{det}(I-K)=-\operatorname{tr}\left((I-K)^{-1} \frac{\partial K}{\partial s}\right) .
$$

By calculus

$$
\frac{\partial K}{\partial s} \doteq-K(x, s) \delta(y-s) .
$$

(If $L$ is an operator with kernel $L(x, y)$ we denote this by $L \doteq L(x, y)$.) Substituting this into the above expression gives

$$
\frac{d}{d s} \log \operatorname{det}(I-K)=-R(s, s)
$$


where $R(x, y)$ is the resolvent kernel of $K$, i.e. $R=(I-K)^{-1} K \doteq R(x, y)$. The resolvent kernel $R(x, y)$ is smooth in $x$ but discontinuous in $y$ at $y=s$. The quantity $R(s, s)$ is interpreted to mean

$$
\lim _{\substack{y \rightarrow s \\ y \in J}} R(s, y) .
$$

\subsubsection{Representation for $R(x, y)$}

If $M$ denotes the multiplication operator, $(M f)(x)=x f(x)$, then

$$
[M, K] \doteq x K(x, y)-K(x, y) y=(x-y) K(x, y)=\varphi(x) \psi(y)-\psi(x) \varphi(y)
$$

As an operator equation this is

$$
[M, K]=\varphi \otimes \psi-\psi \otimes \varphi .
$$

(We define $a \otimes b \doteq a(x) b(y)$ and let $[\cdot, \cdot]$ denote the commutator.) Thus

$$
\begin{aligned}
{\left[M,(I-K)^{-1}\right] } & =(I-K)^{-1}[M, K](I-K)^{-1} \\
& =(I-K)^{-1}(\varphi \otimes \psi-\psi \otimes \varphi)(I-K)^{-1} \\
& =Q \otimes P-P \otimes Q
\end{aligned}
$$

where we have introduced

$$
Q(x ; s)=Q(x)=(I-K)^{-1} \varphi \quad \text { and } \quad P(x ; s)=P(x)=(I-K)^{-1} \psi .
$$

On the other hand since $(I-K)^{-1} \doteq \rho(x, y)=\delta(x-y)+R(x, y)$,

$$
\left[M,(I-K)^{-1}\right] \doteq(x-y) \rho(x, y)=(x-y) R(x, y) .
$$

Comparing (7) and (9) we see that

$$
R(x, y)=\frac{Q(x) P(y)-P(x) Q(y)}{x-y}, \quad x, y \in J .
$$

Taking $y \rightarrow x$ gives

$$
R(x, x)=Q^{\prime}(x) P(x)-P^{\prime}(x) Q(x) .
$$

Introducing

$$
q(s)=Q(s ; s) \text { and } \quad p(s)=P(s ; s),
$$

we have

$$
R(s, s)=Q^{\prime}(s ; s) p(s)-P^{\prime}(s ; s) q(s), \quad s<x, y<\infty .
$$

The reader may have noted that the expression (10) for $R(x, y)$ depends only upon the kernel $K(x, y)$ having the form (11). As far as the authors are aware, the generality of this representation for $R(x, y)$ was first stressed by Its, et al. [23], though it appears in the context of the sine kernel in the work of Jimbo, et al. [26]. 


\subsubsection{Formulas for $Q^{\prime}(x)$ and $P^{\prime}(x)$}

As we just saw, we need expressions for $Q^{\prime}(x)$ and $P^{\prime}(x)$. If $D$ denotes the differentiation operator, $d / d x$, then

$$
\begin{aligned}
Q^{\prime}(x ; s) & =D(I-K)^{-1} \varphi \\
& =(I-K)^{-1} D \varphi+\left[D,(I-K)^{-1}\right] \varphi \\
& =(I-K)^{-1} \psi+\left[D,(I-K)^{-1}\right] \varphi \\
& =P(x)+\left[D,(I-K)^{-1}\right] \varphi .
\end{aligned}
$$

We need the commutator

$$
\left[D,(I-K)^{-1}\right]=(I-K)^{-1}[D, K](I-K)^{-1} .
$$

Integration by parts shows

$$
[D, K] \doteq\left(\frac{\partial K}{\partial x}+\frac{\partial K}{\partial y}\right)+K(x, s) \delta(y-s) .
$$

(The $\delta$ function comes from differentiating the characteristic function $\chi$.) Using the specific form for $\varphi$ and $\psi\left(\varphi^{\prime}=\psi, \psi^{\prime}=x \varphi\right)$ we compute:

$$
\left(\frac{\partial K}{\partial x}+\frac{\partial K}{\partial y}\right)=\varphi(x) \varphi(y) .
$$

Thus

$$
\left[D,(I-K)^{-1}\right] \doteq-Q(x) Q(y)+R(x, s) \rho(s, y) .
$$

(Recall $(I-K)^{-1} \doteq \rho(x, y)$.) We now use this in (14)

$$
\begin{aligned}
Q^{\prime}(x ; s) & =P(x)-Q(x)(Q, \varphi)+R(x, s) q(s) \\
& =P(x)-Q(x) u(s)+R(x, s) q(s)
\end{aligned}
$$

where the inner product $(Q, \varphi)$ is denoted by $u(s)$. Evaluating at $x=s$ gives

$$
Q^{\prime}(s ; s)=p(s)-q(s) u(s)+R(s, s) q(s) .
$$

We now apply the same procedure to compute $P^{\prime}$ encountering the one new feature that since $\psi^{\prime}(x)=x \varphi(x)$ we need to introduce an additional commutator term:

$$
\begin{aligned}
P^{\prime}(x ; s) & =D(I-K)^{-1} \psi \\
& =(I-K)^{-1} D \psi+\left[D,(I-K)^{-1}\right] \psi \\
& =M(I-K)^{-1} \varphi+\left[(I-K)^{-1}, M\right] \varphi+\left[D,(I-K)^{-1}\right] \psi \\
& =x Q(x)+(P \otimes Q-Q \otimes P) \varphi+(-Q \otimes Q) \psi+R(x, s) p(s) \\
& =x Q(x)+P(x)(Q, \varphi)-Q(x)(P, \varphi)-Q(x)(Q, \psi)+R(x, s) p(s) \\
& =x Q(x)-2 Q(x) v(s)+P(x) u(s)+R(x, s) p(s) .
\end{aligned}
$$


Here $v=(P, \varphi)=(\psi, Q)$. Evaluating at $x=s$ gives

$$
P^{\prime}(s ; s)=s q(s)+2 q(s) v(s)+p(s) u(s)+R(s, s) p(s) .
$$

Using this and the expression for $Q^{\prime}(s ; s)$ in 13 gives

$$
R(s, s)=p^{2}-s q^{2}+2 q^{2} v-2 p q u .
$$

\subsubsection{First order equations for $q, p, u$ and $v$}

By the chain rule

$$
\frac{d q}{d s}=\left.\left(\frac{\partial}{\partial x}+\frac{\partial}{\partial s}\right) Q(x ; s)\right|_{x=s} .
$$

We have already computed the partial of $Q(x ; s)$ with respect to $x$. The partial with respect to $s$ is

$$
\begin{aligned}
\frac{\partial Q(x ; s)}{\partial s} & =(I-K)^{-1} \frac{\partial K}{\partial s}(I-K)^{-1} \varphi \\
& =-R(x, s) q(s)
\end{aligned}
$$

where we used (6). Adding the two partial derivatives and evaluating at $x=s$ gives

$$
\frac{d q}{d s}=p-q u
$$

A similar calculation gives

$$
\frac{d p}{d s}=s q-2 q v+p u
$$

We derive first order differential equations for $u$ and $v$ by differentiating the inner products:

$$
\begin{aligned}
u(s) & =\int_{s}^{\infty} \varphi(x) Q(x ; s) d x \\
\frac{d u}{d s} & =-\varphi(s) q(s)+\int_{s}^{\infty} \varphi(x) \frac{\partial Q(x ; s)}{\partial s} d x \\
& =-\left(\varphi(s)+\int_{s}^{\infty} R(s, x) \varphi(x) d x\right) q(s) \\
& =-(I-K)^{-1} \varphi(s) q(s) \\
& =-q^{2} .
\end{aligned}
$$

Similarly,

$$
\frac{d v}{d s}=-p q
$$

\subsubsection{Painlevé II and $F_{2}$}

From the first order differential equations for $q, u$ and $v$ it follows immediately that the derivative of $u^{2}-2 v-q^{2}$ is zero. Examining the behavior near $s=\infty$ to check that the constant of integration is zero then gives

$$
u^{2}-2 v=q^{2},
$$


a first integral. We now differentiate (19) with respect to $s$, use the first order differential equations for $p$ and $u$, and then the first integral to deduce that $q$ satisfies the Painlevé $I I$ equation

$$
q^{\prime \prime}=s q+2 q^{3} .
$$

Checking the asymptotics of the Fredholm determinant $\operatorname{det}(I-K)$ for large $s$ shows we want the solution to the Painlevé II equation with boundary condition

$$
q(s) \sim \operatorname{Ai}(s) \text { as } s \rightarrow \infty .
$$

That a solution $q$ exists and is unique follows from the representation of the Fredholm determinant in terms of it. Independent proofs of this, as well as the asymptotics as $s \rightarrow \infty$ were given by [22, 11, 14].

Since the kernel of $\left[D,(I-K)^{-1}\right]$ is $(\partial / \partial x+\partial / \partial y) R(x, y)$, (15) says

$$
\left(\frac{\partial}{\partial x}+\frac{\partial}{\partial y}\right) R(x, y)=-Q(x) Q(y)+R(x, s) \rho(s, y) .
$$

In computing $\partial Q(x ; s) / \partial s$ we showed that

$$
\frac{\partial}{\partial s}(I-K)^{-1} \doteq \frac{\partial}{\partial s} R(x, y)=-R(x, s) \rho(s, y) .
$$

Adding these two expressions,

$$
\left(\frac{\partial}{\partial x}+\frac{\partial}{\partial y}+\frac{\partial}{\partial s}\right) R(x, y)=-Q(x) Q(y)
$$

and then evaluating at $x=y=s$ gives

$$
\frac{d}{d s} R(s, s)=-q^{2}
$$

Integration (and recalling (5) gives,

$$
\frac{d}{d s} \log \operatorname{det}(I-K)=-\int_{s}^{\infty} q^{2}(x) d x ;
$$

and hence,

$$
\log \operatorname{det}(I-K)=-\int_{s}^{\infty}\left(\int_{y}^{\infty} q^{2}(x) d x\right) d y=-\int_{s}^{\infty}(x-s) q^{2}(x) d x .
$$

To summarize, we have shown that the distribution function $F_{2}$ has the Painlevé representation

$$
F_{2}(s)=\exp \left(-\int_{s}^{\infty}(x-s) q^{2}(x) d x\right)
$$

where $q$ satifies the Painlevé II equation (21) subject to the boundary condition (22).

Alternatively, it is easy to check that (23) integrates to

$$
R(s, s)=\left(q^{\prime}\right)^{2}-s q^{2}-q^{4} .
$$

This and together with (21) shows that $R(s, s)$ itself satisfies

$$
\left(R^{\prime \prime}\right)^{2}+4 R^{\prime}\left(\left(R^{\prime}\right)^{2}-s R^{\prime}+R\right)=0 .
$$

This last differential equation is the Jimbo-Miwa-Okamoto [27, 36] $\sigma$ form for Painlevé II. (Observe that (25) and (17) give another identity between $q, p, u$ and $v$.) 


\subsection{Remarks}

1. In 443 the general case $J=\bigcup\left(a_{2 j-1}, a_{2 j}\right)$ is first considered and then specialized to $J=(s, \infty)$. Here we have simply given the proof of 43 ] for this special case from the very beginning.

2. The asymptotics of $F_{2}(s)$ as $s \rightarrow-\infty$ are important and they require the solution of a connection problem for Painlevé II. This connection problem is solved in [22, 11, 14] and applied in [43] to $F_{2}$ with the result that

$$
F_{2}(s) \sim \frac{\tau_{0}}{(-s)^{1 / 8}} e^{s^{3} / 12}, \quad s \rightarrow-\infty .
$$

The constant $\tau_{0}$ is conjectured to equal $e^{\zeta^{\prime}(-1)} 2^{1 / 24}$.

3. For the distribution of the next-largest, next-next-largest, etc. eigenvalues, see 433.

4. The connection between certain classes of Fredholm determinants and integrable systems of the Painlevé type begins with work on the two-dimensional Ising model 53, 32]. In this model the spin-spin correlation functions in the scaling limit are expressed in terms of a third Painlevé transcendent. Painlevé functions first appear in random matrix theory in [26] where the Fredholm determinant of the sine kernel, $J=(-t, t)$, is given in terms of a fifth Painlevé transcendent.

5. In 14 a general theory was developed that relates Fredholm determinants of operators $K$ with kernel of the form (11) acting on $J=\bigcup\left(a_{2 j-1}, a_{2 j}\right)$ with integrable systems. This theory assumes that the functions $\varphi$ and $\psi$ satisfy a first order linear system of differential equations with rational coefficients. For related developments see [1, 21, [38, 52].

\section{Orthogonal and Symplectic Ensembles}

The probabilities, $E_{\beta}(0 ; J)$, that a set $J$ consisting of a finite union of open intervals contains no eigenvalues for the finite $N \operatorname{GOE}(\beta=1)$ and $\operatorname{GSE}(\beta=4)$ are more difficult to compute than are the probabilities for the finite $N$ GUE $(\beta=2)$. The essential problem comes down to the fact that we must deal with Fredholm determinants of operators with matrix-valued kernels 457 .

Changing notation slightly, we denote by $S$ the operator $K_{N, 2}$ of the previous section and let $\varphi$ and $\psi$ be as before; namely, (2) and (3), respectively. Let $\varepsilon$ equal the operator with kernel $\varepsilon(x-y)=\frac{1}{2} \operatorname{sgn}(x-y)$ (sgn is the sign function) and $D$ the differentiation operator. It then follows from Mehta's representation [34] of the $n$-point correlations [15, 46] and for $N$ even (this case is slightly simpler) that

$$
E_{N, 1}(0 ; J)^{2}=\operatorname{det}\left(I-K_{N, 1}\right)
$$

where

$$
K_{N, 1}=\chi\left(\begin{array}{cc}
S+\psi \otimes \varepsilon \varphi & S D-\psi \otimes \varphi \\
\varepsilon S-\varepsilon+\varepsilon \psi \otimes \varepsilon \varphi & S+\varepsilon \varphi \otimes \psi
\end{array}\right) \chi
$$

and $\chi$ is the operator of multiplication by $\chi_{J}(x)$, the characteristic function of $J$. There is a similar representation for $E_{N, 4}(0 ; J)$ [45, 46]. This form for $K_{N, 1}$ indicates our change of 
view; namely, we think of $K_{N, 1}$ as a $2 \times 2$ matrix with operator entries. We then manipulate these determinants of these operators to put them in the form

$$
E_{N, \beta}(0 ; J)^{2}=E_{N, 2}(0 ; J) \operatorname{det}\left(I-\sum_{k=1}^{n} \alpha_{k} \otimes \beta_{k}\right)
$$

for $\beta=1$, 4. (Of course, the $\alpha_{k}$ 's and $\beta_{k}$ 's depend upon the ensemble.) The last determinant is evaluated using the general formula [18]

$$
\operatorname{det}\left(I-\sum_{k=1}^{n} \alpha_{k} \otimes \beta_{k}\right)=\operatorname{det}\left(\delta_{j, k}-\left(\alpha_{j}, \beta_{k}\right)\right)_{j, k=1, \ldots, n}
$$

where $\left(\alpha_{j}, \beta_{k}\right)$ denotes the inner product.

We then derive differential equations for the inner products introduced by the last determinant. For $J=(t, \infty), E_{\beta}(0 ; J)$ is again the distribution function of the largest eigenvalue, $F_{N, \beta}(t)$, in either the $\operatorname{GOE}(\beta=1)$ or $\operatorname{GSE}(\beta=4)$. Again we take the edge scaling limit:

$$
F_{\beta}(s):=\lim _{N \rightarrow \infty} F_{N, \beta}\left(2 \sigma \sqrt{N}+\frac{\sigma s}{N^{1 / 6}}\right) .
$$

Here $\sigma$ is the standard deviation of the Gaussian distribution on the off-diagonal matrix elements. Our choice corresponds to $\sigma=1 / \sqrt{2}$, but as the notation suggests, $F_{\beta}(s)$ is independent of $\sigma$. Our final results are

$$
\begin{aligned}
F_{1}(s)^{2} & =F_{2}(s) \exp \left(-\int_{s}^{\infty} q(x) d x\right), \\
F_{4}(s / \sqrt{s})^{2} & =F_{2}(s) \cosh ^{2}\left(\frac{1}{2} \int_{s}^{\infty} q(x) d x\right)
\end{aligned}
$$

and $F_{2}$ and $q$ are as before.

\subsection{Remarks}

1. Strictly speaking these distributions are only applicable in the limit that the size of the matrices, $N$, tends to infinity. In practice they are good approximations once $N \geq 200$. Some comparisons with finite $N$ simulations are in [47].

2. Table 1 displays some statistics of $F_{\beta}$ and Figure 1 graphs the densities $f_{\beta}(s)=$ $d F_{\beta} / d s$.

3. As has been established for the edge scaling limit of unitary matrix ensembles [8, 13, 41], we expect the distributions $F_{1}$ and $F_{4}$ to be the generic distributions at the edge of the spectrum (no "fine tuning" of the potential) for a large class of orthogonal and symplectic matrix ensembles. This universality has been proved for the orthogonal and symplectic ensembles in the case of a quartic potential [42]. In [30] the distribution of the appropriately centered and normalized largest eigenvalue of a $p$ variate Wishart distribution on $n$ degrees of freedom is shown to converge, as $n \rightarrow \infty, p \rightarrow \infty$, such that $n / p$ is fixed, to $F_{1}$. 
Table 1: The mean $\left(\mu_{\beta}\right)$, standard deviation $\left(\sigma_{\beta}\right)$, skewness $\left(S_{\beta}\right)$ and kurtosis $\left(K_{\beta}\right)$ of $F_{\beta}$.

\begin{tabular}{|c|cccc|}
\hline$\beta$ & $\mu_{\beta}$ & $\sigma_{\beta}$ & $S_{\beta}$ & $K_{\beta}$ \\
\hline 1 & -1.20653 & 1.2680 & 0.293 & 0.165 \\
2 & -1.77109 & 0.9018 & 0.224 & 0.093 \\
4 & -2.30688 & 0.7195 & 0.166 & 0.050 \\
\hline
\end{tabular}

4. The past few years has seen an explosion of activity connecting random matrix theory and combinatorial problems related to the Robinson-Schensted-Knuth algorithm. At the level of limit laws, this began with the work of Baik, Deift and Johansson [4] (for a review of this work, see [12]) who discovered that $F_{2}$ is the limiting distribution of the (appropriately centered and normalized) length of the longest increasing subsequence of a random permutation. For related developments see [2, 3, 5, 6, 7, 9, 19, 20, 24, $25,28,29,31,37,39,40,48,49,50$.

\section{Acknowledgements}

The first author thanks Oleg Zaboronski and Alan Newell at the Mathematics Institute, University of Warwick, and John Harnad and Alexander Its at the CRM Workshop Isomondromic Deformations and Applications for their kind hospitality. This work was supported in part by the National Science Foundation through grants DMS-9802122 (first author) and DMS-9732687 (second author). 
Figure 1: The probability density $f_{\beta}$ of the largest eigenvalue, $\beta=1,2,4$.

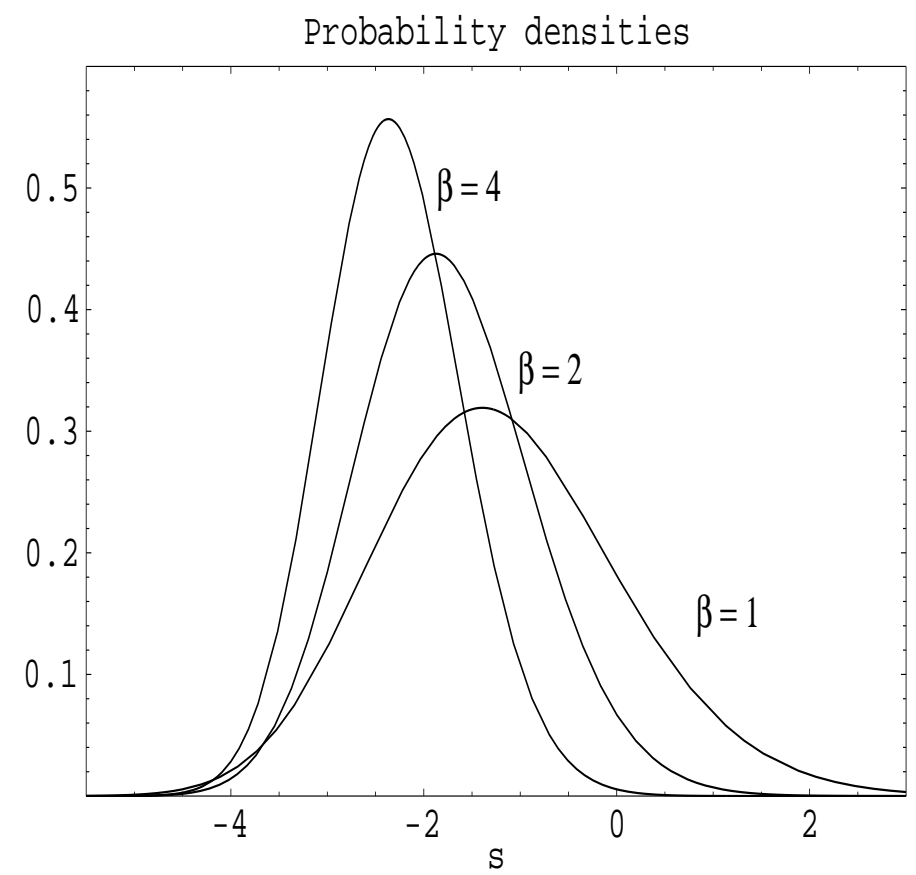




\section{References}

[1] M. Adler, T. Shiota and P. van Moerbeke, Random matrices, Virasoro algebras and noncommutative KP, Duke Math. J. 94 (1998), 379-431.

[2] M. Adler and P. van Moerbeke, Integrals over classical groups, random permutations, Toda and Toeplitz lattices, preprint (arXiv: math.CO/9912143).

[3] D. Aldous and P. Diaconis, Longest increasing subsequences: From patience sorting to the Baik-Deift-Johansson theorem, Bull. Amer. Math. Soc. 36 (1999), 413-432.

[4] J. Baik, P. Deift and K. Johansson, On the distribution of the length of the longest increasing subsequence of random permutations, J. Amer. Math. Soc. 12 (1999), 11191178.

[5] J. Baik and E. Rains, The asymptotics of monotone subsequences of involutions, preprint (arXiv: math.CO/9905084).

[6] J. Baik and E. Rains, Symmetrized random permutations, preprint (arXiv: math.CO/9910019).

[7] J. Baik and E. Rains, Limiting distributions for a polynuclear growth model with external sources, J. Statist. Phys. 100 (2000), 523-541.

[8] P. Bleher and A. Its, Semiclassical asymptotics of orthogonal polynomials, RiemannHilbert problem, and universality in the matrix model, Ann. Math. 150 (1999), 185-266.

[9] A. Borodin, A. Okounkov and G. Olshanski, Asymptotics of Plancherel measures for symmetric groups, J. Amer. Math. Soc. 13 (2000), 481-515.

[10] M. J. Bowick and E. Brézin, Universal scaling of the tail of the density of eigenvalues in random matrix models, Phys. Letts. B268 (1991) 21-28.

[11] P. A. Clarkson and J. B. McLeod, A connection formula for the second Painlevé transcendent, Arch. Rat. Mech. Anal. 103 (1988) 97-138.

[12] P. A. Deift, Integrable systems and combinatorial theory, Notices Amer. Math. Soc. 47 (2000), 631-640.

[13] P. Deift, T. Kriecherbauer, K. T-R McLaughlin, S. Venakides and X. Zhou, Uniform asymptotics for polynomials orthogonal with respect to varying exponential weight and applications to universality questions in random matrix theory, Commun. Pure Appl. Math. 52 (1999), 1335-1425.

[14] P. A. Deift and X. Zhou, Asymptotics for the Painlevé II equation, Commun. Pure Appl. Math. 48 (1995) 277-337.

[15] F. J. Dyson, Correlations between eigenvalues of a random matrix, Commun. Math. Phys. 19 (1970) 235-250.

[16] P. J. Forrester, The spectrum edge of random matrix ensembles, Nucl. Phys. B402 (1993) 709-728.

[17] M. Gaudin, Sur la dlo limite de l'espacement des valeurs propres d'une matrice aléatoire, Nucl. Phys. 25 (1961) 447-458. (Reprinted in C. E. Porter, Statistical Theories of Spectra: Fluctuations, Academic Press, New York, 1965.)

[18] I. C. Gohberg and M. G. Krein, Introduction to the Theory of Linear Nonselfadjoint Operators, Transl. Math. Monogr. 35, Providence, RI: Amer. Math. Soc. 1969. 
[19] J. Gravner, C. A. Tracy and H. Widom, Limit theorems for height fluctuations in a class of discrete space and time growth models, preprint (arXiv: math.PR/0005133).

[20] J. Gravner, C. A. Tracy and H. Widom, A growth model in a random environment, preprint (arXiv: math.PR/0011150).

[21] J. Harnad and A. R. Its, Integrable Fredholm operators and dual isomonodromic deformations, preprint (arXiv: nlin.SI/9706002).

[22] S. P. Hastings and J. B. McLeod, A boundary value problem associated with the second Painlevé transcendent and the Korteweg de Vries equation, Arch. Rat. Mech. Anal. 73 (1980) 31-51.

[23] A. R. Its, A. G. Izergin, V. E. Korepin, and N. A. Slavnov, Differential equations for quantum correlations, Int. J. Mod. Physics B4 (1990) 1003-1037.

[24] A. R. Its, C. A. Tracy and H. Widom, Random words, Toeplitz determinants and integrable systems. I, preprint (arXiv: math.CO/9909169).

[25] A. R. Its, C. A. Tracy and H. Widom, Random words, Toeplitz determinants and integrable systems. II, preprint (arXiv: nlin.SI/0004018).

[26] M. Jimbo, T. Miwa, Y. Môri, and M. Sato, Density matrix of an impenetrable Bose gas and the fifth Painlevé transcendent, Physica 1D (1980) 80-158.

[27] M. Jimbo and T. Miwa, Monodromy preserving deformations of linear ordinary differential equations with rational coefficients. II, Physica 2D (1981) 407-448.

[28] K. Johansson, Shape fluctuations and random matrices, Commun. Math. Phys. 209 (2000), 437-476.

[29] K. Johansson, Discrete orthogonal polynomial ensembles and the Plancherel measure, preprint (arXiv: math.CO/9906120).

[30] I. M. Johnstone, On the distribution of the largest principal component, preprint.

[31] G. Kuperberg, Random words, quantum statistics, central limits, random matrices, preprint (arXiv: math.PR/9909104).

[32] B. M. McCoy, C. A. Tracy and T. T. Wu, Painlevé functions of the third kind, J. Math. Phys. 18 (1977) 1058-1092.

[33] M. L. Mehta, On the statistical properties of the level-spacings in nuclear spectra, Nucl. Phys. 18 (1960) 395-419. (Reprinted in C. E. Porter, Statistical Theories of Spectra: Fluctuations, Academic Press, New York, 1965.)

[34] M. L. Mehta, A note on correlations between eigenvalues of a random matrix, Commun. Math. Phys. 20 (1971) 245-250.

[35] M. L. Mehta, Random Matrices, Academic Press, San Diego, 1991.

[36] K. Okamoto, Studies on the Painlevé equations III. Second and Fourth Painlevé equations, $P_{I I}$ and $P_{I V}$, Math. Ann. 275 (1986) 221-255.

[37] A. Okounkov, Random matrics and random permutations, preprint (arXiv: math.CO/9903176).

[38] J. Palmer, Deformation analysis of matrix models, Physica D 78 (1994), 166-185.

[39] M. Prähofer and H. Spohn, Statistical self-similarity of one-dimensional growth processes, Physica A 279 (2000), 342-352. 
[40] M. Prähofer and H. Spohn, Universal distributions for growth processes in $1+1$ dimensions and random matrices, Phys. Rev. Letts. 84 (2000), 4882-4885.

[41] A. Soshnikov, Universality at the edge of the spectrum in Wigner random matrices, Commun. Math. Phys. 207 (1999), 697-733.

[42] A. Stojanovic, Universality in orthogonal and symplectic invariant matrix models with quartic potential, preprint.

[43] C. A. Tracy and H. Widom, Level-spacing distributions and the Airy kernel, Commun. Math. Phys., 159 (1994) 151-174.

[44] C. A. Tracy and H. Widom, Fredholm determinants, differential equations and matrix models, Commun. Math. Phys. 163 (1994) 33-72.

[45] C. A. Tracy and H. Widom, On orthogonal and symplectic matrix ensembles, Commun. Math. Phys. 177 (1996) 727-754.

[46] C. A. Tracy and H. Widom, Correlation functions, cluster functions, and spacing distributions for random matrices, J. Statist. Phys. 92 (1998) 809-835.

[47] C. A. Tracy and H. Widom, Distribution of the largest eigenvalue in the gaussian ensembles: $\beta=1,2,4$, in Calogero-Sutherland-Moser Models, eds. J. F. van Diejen and L. Vinet, CRM Series in Mathematical Physics 4, Springer-Verlag, New York, 2000, pp. $461-472$.

[48] C. A. Tracy and H. Widom, Random unitary matrices, permutations and Painlevé, Commun. Math. Phys. 207 (1999), 665-685.

[49] C. A. Tracy and H. Widom, On the distribution of the lengths of the longest monotone subsequences in random words, preprint (arXiv: math.CO/9904042).

[50] P. van Moerbeke, Integrable lattices: Random matrices and random permutations, preprint (arXiv: math.CO/0010135).

[51] H. Widom, On the relation between orthogonal, symplectic and unitary matrix models, J. Statist. Phys. 94 (1999), 347-363.

[52] N. S. Witte, P. J. Forrester and C. M. Cosgrove, Integrability, random matrices and Painlevé transcendents, preprint (arXiv: math-ph/0008033).

[53] T. T. Wu, B. M. McCoy, C. A. Tracy and E. Barouch, Spin-spin correlation functions of the two-dimensional Ising model: Exact theory in the scaling region, Phys. Rev. B13 (1976) 316-374. 